Nervenarzt 2022 $\cdot 93: 835-837$

https://doi.org/10.1007/s00115-021-01259-x

Angenommen: 26. November 2021

Online publiziert: 13. Januar 2022

(C) The Author(s), under exclusive licence to Springer Medizin Verlag GmbH, ein Teil von Springer Nature 2022, korrigierte Publikation 2022

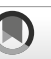

Check for
updates

\section{Die Borna-Virus-Enzephalitis als Differenzialdiagnose zur seronegativen Autoimmunenzephalitis}

\author{
H. Meier ${ }^{1} \cdot$ C. Bauer ${ }^{1} \cdot$ W. Finkenzeller ${ }^{1} \cdot$ J. Nentwich ${ }^{2} \cdot$ M. Städt ${ }^{3} \cdot$ P. Steininger ${ }^{4}$. \\ K. Korn ${ }^{4} \cdot$ A. Ensser ${ }^{4} \cdot$ F. Erbguth ${ }^{1}$ \\ ${ }^{1}$ Klinik für Neurologie, Universitätsklinik bzw. Universitätsinstitut der Paracelsus Medizinischen \\ Privatuniversität, Klinikum Nürnberg, Nürnberg, Deutschland; ${ }^{2}$ Klinik für Innere Medizin, \\ Universitätsklinik bzw. Universitätsinstitut der Paracelsus Medizinischen Privatuniversität, Klinikum \\ Nürnberg, Nürnberg, Deutschland; ${ }^{3}$ Klinik für Neuroradiologie, Universitätsklinik bzw. Universitätsinstitut \\ der Paracelsus Medizinischen Privatuniversität, Klinikum Nürnberg, Nürnberg, Deutschland; \\ ${ }^{4}$ Virologisches Institut, Universitätsklinikum Erlangen, Friedrich-Alexander-Universität Erlangen- \\ Nürnberg, Erlangen, Deutschland
}

\section{Hintergrund}

Die meist letale Borna-Virus-Enzephalitis (BVE) wird durch das "Borna disease virus 1" (BoDV-1) und "variegated squirrel bornavirus 1" (VSBV-1) verursacht [2]. Die aktuelle Inzidenz der Borna-Virus-Enzephalitis in Deutschland beträgt ca. 2 Infektionen/ Jahr, meist in endemischen Gebieten im Südosten Deutschlands [2, 5]. Wir möchten über zwei Erkrankungsfälle berichten, die klinisch zunächst als seronegative $\mathrm{Au}$ toimmunenzephalitiden (AE) interpretiert wurden, sich jedoch beide als eine BVE herausstellten.

\section{Fallbeispiel 1}

Die 32-jährige Patientin wurde im Februar 2021 aus einem Krankenhaus der Grundversorgung intubiert übernommen, wo sie wegen Diarrhöen, Fieber und Gliederschmerzen aufgenommen worden war und innerhalb einer Woche eine Dysphagie und Vigilanzminderung entwickelte. Die Liquoranalyse ergab unauffällige Notfallwerte mit einer negativen Multiplex$P C R$, das CMRT war unauffällig.

Eine Liquorkontrolle erbrachte eine leichte lymphozytäre Pleozytose $(23 / \mu \mathrm{l})$ bei ansonsten normalen Parametern. Die Untersuchungen auf Antikörper in Serum und Liquor waren negativ (onkoneural, GQ1b, GM1 und GM2, Glutamat-Decar- boxylase, LGI1, AMPA1, AMPA2, GABA, NMDA, Kaliumkanalkomplex). Unauffällig waren auch ein Vaskulitis- und immunologisches Screening, die Infektionsserologie und eine Porphyriediagnostik.

Im Verlauf zeigte die Patientin fokale klonische Anfälle des rechten Armes, weshalb eine antiepileptische Therapie begonnen wurde.

Unter der Arbeitsdiagnose einer seronegativen $A E$ wurde eine Therapie mit Prednisolon $(1000 \mathrm{mg})$ über 5 Tage etabliert und um eine Plasmapherese über 5 Tage ergänzt.

Zunächst stabilisierte sich der Zustand der Patientin, sodass sie 2 Wochen nach Zuverlegung in wachem Zustand ohne motorisches Defizit extubiert werden konnte. Die klinische Besserung wurde als ein Ansprechen auf die Therapie und als Bestätigung der Verdachtsdiagnose gewertet. Wegen einer persistierenden ausgeprägten Dysphagie und respiratorischer Verschlechterung musste sie jedoch reintubiert werden.

Trotzerneuter Plasmapherese über $7 \mathrm{Ta}-$ ge und nachfolgender immunsuppressiver Therapie mit Rituximab (2-mal je 1000 mg) und Cyclophosphamid (2-mal je $1050 \mathrm{mg}$ ) verschlechterte sich der Zustand der Patientin bis hin zum Koma.

Nach 6 Wochen zeigte eine erneute CMRT eine deutliche Befundverschlechterung mit Nachweis fleckiger symme- 


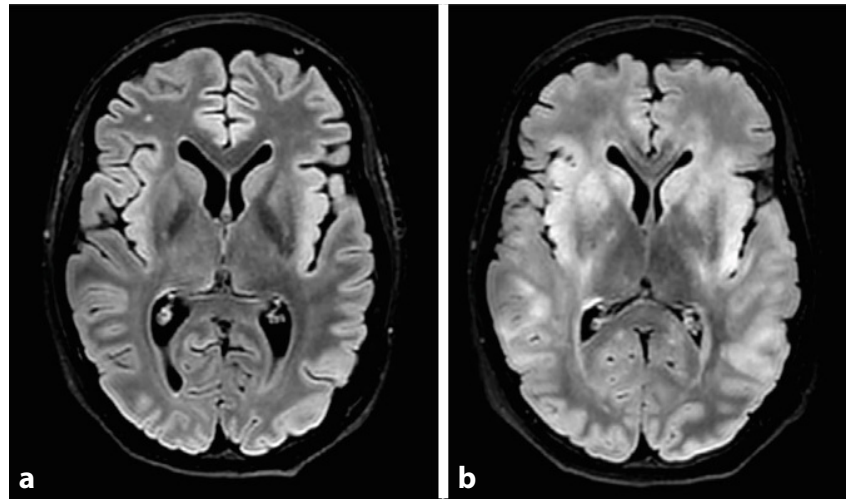

Abb. 1 \ Fleckige Hyperintensitäten im frontotemporoparietalen Rindenbereich beidseits in FLAIR/T2 mit Einbeziehung der Basalganglien und Aussparung des Thalamus. a, b Transversale FLAIR-gewichtete cMRT-Schnittbilder

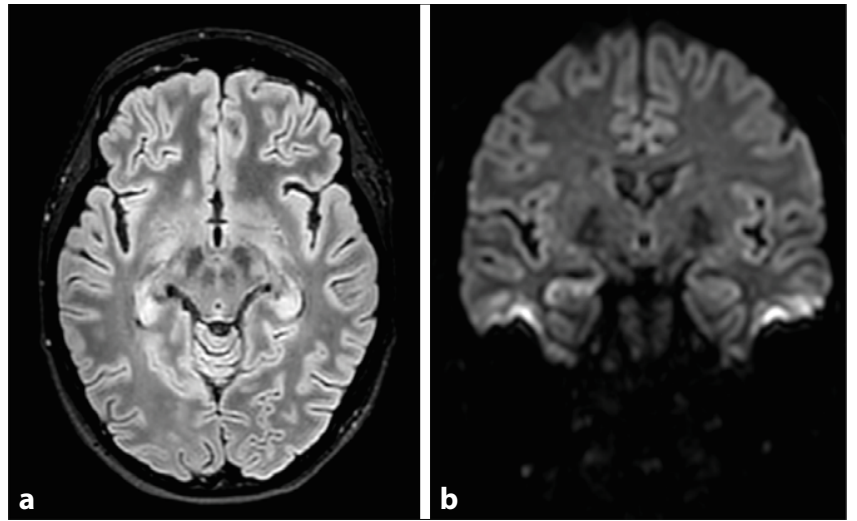

Abb. $2 \Delta$ a Ödemäquivalente Signalalterationen der Hippocampi beidseits in FLAIR/T2. b Diffusionsgewichtete koronare Sequenz mit rechtsbetonter Diffusionsstörung der Hippocampi trischer kontrastmittelaufnehmender $\mathrm{Hy}$ perintensitäten (T2/Flair) frontotemporoparietal beidseits unter Einbeziehung der Basalganglien und Aussparung des Thalamus (- Abb. 1). Zur weiteren diagnostischen Einordnung wurde eine Hirnbiopsie durchgeführt, die zunächst eine lymphozytär vermittelte Enzephalitis bestätigte. Daraufhin wurde unter der weiteren Annahme einer AE Bortezomib verabreicht.

Eine erneute Untersuchung (Liquor, Serum, Hirnbiopsat) unter Einbeziehung des Borna-Virus ergab einen eindeutig positiven Befund auf BoDV-1 im Biopsiematerial und einen grenzwertig positiven PCR-Befund im Liquor (CT-Wert 37).

Nach Beendigung der immunsuppressiven Therapien wurde eine antivirale Therapie mit Favipiravir begonnen, wodurch es zu keiner klinischen Besserung kam. Im Rahmen eines palliativen Therapiezielwechsels verstarb die Patientin nach insgesamt 10 Wochen stationären Aufenthaltes in unserer Klinik.

Ein Kontakt zu potenziell mit BoDV-1 infizierten Tieren konnte retrospektiv nicht eruiert werden, letztlich bleibt der Übertragungsweg bei der Patientin unklar.

\section{Fallbeispiel 2}

Die 23-jährige Patientin wurde im April 2021 mit Kopfschmerzen, subfebrilen Temperaturen und einem erstmaligen generalisierten epileptischen Anfall stationär aufgenommen.
Die Liquoruntersuchung erbrachte eine leichte Pleozytose $(49 / \mu l)$ bei sonst unauffälligen Notfallwerten. Ein aussagekräftiges cMRT war trotz Sedierung nicht möglich.

Bei zunehmender Agitation und Halluzinationen wurde unter dem Verdacht einer AE neben Levetiracetam eine hochdosierte Steroidpulstherapie mit Prednisolon über 5 Tage (500 und $1000 \mathrm{mg}$ ) durchgeführt, gefolgt von einer IVIG-Therapie (je $40 \mathrm{~g}$ ) über 3 Tage.

Fünf Tage nach Aufnahme führten erneute generalisierte epileptische Anfälle und eine zunehmende Vigilanzminderung zur maschinellen Beatmung auf der Intensivstation. Die AE-Antiköperbestimmungen im Liquor und Serum waren negativ.

Nach erfolgloser Plasmapherese (7 Tage) entwickelte die komatöse Patientin eine zunehmende Störung der Pupillomotorik sowie der Temperaturregulation als Hinweis auf eine Hirnstammaffektion. Im cMRT zeigten sich ödematöse Veränderungen im Bereich der Hippocampi beidseits mit einer rechtsbetonten Diffusionsstörung, kompatibel mit der Verdachtsdiagnose einer AE (• Abb. 2).

Nach einer Therapieeskalation mittels Cyclophosphamid ( $900 \mathrm{mg}$ ) und Rituximab (1000 mg) wurde 14Tage nach Einlieferung in unsere Klinik der Nachweis von BoDV1-RNA im eingesandten Liquor bestätigt, der Serumbefund war negativ.

Bei anhaltend ausgefallenen Hirnstammreflexen, einem Burst-suppressionMuster im EEG sowie einem patholo- gischen Apnoetest entschieden wir uns aufgrund der infausten Prognose für eine Beendigung der intensivmedizinischen Maßnahmen. Die Patientin verstarb 16 Tage nach der Einlieferung in unsere Klinik.

Eine postmortale Hirnbiopsie ergab eine hohe Borna-Virus-Last in dem eingesandten Punktat.

Rückblickend ließ sich eruieren, dass die Katze der Patientin 2 Monate vor Symptombeginn eine Spitzmaus apportiert hatte. Die Patientin selbst hatte keinen direkten Kontakt zu der Maus. Dieses Ereignis wurde retrospektiv als vermutlicher Infektionsweg gewertet.

\section{Diskussion}

In beiden Fällen wurde initial von einer seronegativen $\mathrm{AE}$ ausgegangen, da typische Charakteristika wie psychiatrische Symptome, Bewusstseinsstörungen, epileptische Anfälle und eine Liquorpleozytose sowie im ersten Fall zunächst eine vermeintliche Responsivität auf die initiale immunsuppressive Therapie vorlagen. Zudem zeigten sich im zweiten Fall auch zu einer AE passende T2-Hyperintensitäten im Hyopthalamus beidseits ([4]; • Tab. 1).

Eine frühe - notfalls eskalative - immunsuppressive Therapie bei einer AE gehört zu den wichtigsten Prognosefaktoren [1], weshalb bei unseren Patientinnen bei rascher klinischer Verschlechterung therapeutisch eskaliert wurde.

Die Fälle verdeutlichen die hohe Variabilität des Verlaufes einer BVE und den Stellenwert frühzeitiger diagnostischer Schrit- 
Tab. 1 Diagnostische Kriterien für eine mögliche Autoimmunenzephalitis

Subakute, rasch progrediente Kurzzeitgedächtnisstörung ( $<3$ Monate)

Oder

Qualitative/quantitative Bewusstseinsstörung

Oder

Psychiatrische Symptome

te in Endemiegebieten, auch wenn letztlich ein unauffälliger Liquorbefund keinen sicheren Ausschluss einer BVE bedeutet. Im Zweifelsfall sollte bei einer negativen PCR im Liquor eine Diagnostik mittels einer Hirnbiopsie ergänzt werden.

Bildmorphologisch charakteristisch für die BVE sind T2-Hyperintensitäten im Nucleus caudatus, der Insel und im limbischen System - nie jedoch temporal oder okzipital [3].

Eine zugelassene Therapie der BVE gibt es zum aktuellen Zeitpunkt nicht, ob die in In-vitro-Studien nachgewiesene Wirksamkeit von Favipiravir und Ribavirin eine klinische Relevanz hat, bleibt zum aktuellen Zeitpunkt unklar [6].

\section{Fazit für die Praxis}

- Die BVE ist eine seltene endemisch auftretende Erkrankung, die im klinischen Bild einer AE ähneln kann.

- Bei Verdacht auf eine AE sollte frühzeitig auch an die Borna-Virus-Infektion gedacht und die entsprechenden diagnostischen Schritte eingeleitet werden.

- Die Diagnose wird durch den Nachweis von BoDV-1-RNA mittels PCR im Liquor, Serum und/oder Hirnbiopsat gestellt.

- Bei der Diagnose einer BVE sollte eine Meldung bei dem zuständigen Kompetenzzentrum des Robert Koch-Institutes für Krankheiten durch hochpathogene Erreger erfolgen.

\section{Literatur}

1. Ariño $H$, Armangué $T$, Petit-Pedrol $M$ et al (2016) Anti-LGI1-associated cognitive impairment: Presentation and long-term outcome. Neurology 87:759-765

2. Eisermann P, Rubbenstroth D, Cadar D et al (2021) Active case finding of current bornavirus infections in human encephalitis cases of unknown etiology, Germany, 2018-2020. Emerg Infect Dis J 27:1371

3. Finck T, Liesche-Starnecker F, Probst M et al (2020) Bornavirus encephalitis shows a characteristic magnetic resonance phenotype in humans. Ann Neurol 88:723-735

\section{Korrespondenzadresse}

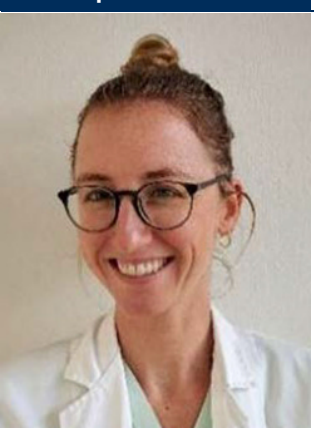

\section{Dr. med. H. Meier}

Klinik für Neurologie, Universitätsklinik bzw. Universitätsinstitut der Paracelsus Medizinischen Privatuniversität, Klinikum Nürnberg

Breslauer Str. 201, 90471 Nürnberg,

Deutschland

helen.meier@klinikum-nuernberg.de

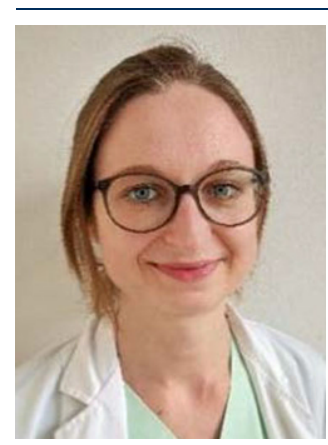

\section{Dr. med. C. Bauer}

Klinik für Neurologie, Universitätsklinik bzw. Universitätsinstitut der Paracelsus Medizinischen Privatuniversität, Klinikum Nürnberg

Breslauer Str. 201, 90471 Nürnberg,

Deutschland

christine.bauer2@klinikum-nuernberg.de

Interessenkonflikt. H. Meier, C. Bauer, W. Finkenzeller, J. Nentwich, M. Städt, P. Steininger, K. Korn, A. Ensser und F. Erbguth geben an, dass kein Interessenkonflikt besteht.
4. Ismail I, Alnaser F, Al-Hashel JY (2021) Seronegative limbic encephalitis manifesting as subacute amnestic syndrome: a case report and review of the literature. J Med Case Rep 15:130

5. Korn K, Coras R, Bobinger T et al (2018) Fatal encephalitis associated with Borna disease virus 1. NEngl J Med 379:1375-1377

6. Tokunaga T, Yamamoto $Y$, Sakai M et al (2017) Antiviral activity of favipiravir (T-705) against mammalian and avian bornaviruses. Antiviral Res 143:237-245 